\title{
EXPERIMENTAL COMPARISON OF MULTI-SHARPENING METHODS APPLIED TO SENTINEL-2 MSI AND SENTINEL-3 OLCI IMAGES
}

\author{
Ahed Alboody ${ }^{1}$, Matthieu Puigt ${ }^{1}$, Gilles Roussel ${ }^{1}$, Vincent Vantrepotte ${ }^{2}$, Cédric Jamet ${ }^{2}$, and Trung Kien Tran ${ }^{2}$ \\ ${ }^{1}$ Univ. Littoral Côte d'Opale, LISIC - EA 4491, F-62219 Longuenesse, France \\ ${ }^{2}$ Univ. Littoral Côte d'Opale, CNRS, LOG - UMR 8187, F-62930 Wimereux, France
}

\begin{abstract}
Multi-spectral images are crucial to detect and to understand phenomena in marine observation. However, in coastal areas, these phenomena are complex and their analyze requires multi-spectral images with both a high spatial and spectral resolution. Unfortunately, no satellite is able to provide both at the same time. As a consequence, multi-sharpening techniques-a.k.a. fusion or superresolution of multi-spectral and/or hyper-spectral images-were proposed and consist of combining information from at least two multi-spectral images with different spatial and spectral resolutions. The fused image then combines their best characteristics. Various methods-based on different strategies and tools-have been proposed to solve this problem. This article presents a comparative review of fusion methods applied to Sentinel-2 MSI (13 spectral bands with a spatial resolution ranging from 10 to $60 \mathrm{~m}$ ) and Sentinel-3 OLCI (21 spectral bands with a spatial resolution of $300 \mathrm{~m}$ ) images. Indeed, both satellites are extensively used in marine observation and, to the best of the authors' knowledge, the fusion of their data was partially investigated (and not in the way we aim to do in this paper). To that end, we provide both a quantitative analysis of the performance of some state-of-the-art methods on simulated images, and a qualitative analysis on real images.
\end{abstract}

Index Terms - Image fusion, Remote sensing, Sentinel-2 MSI, Sentinel-3 OLCI, Simulations, Real data

\section{INTRODUCTION}

The satellite observation of our planet knew significant instrumental advances for several decades, with consequent developments in terms of spatial resolution-e.g., in water remote sensing with high spatial resolution (10-60 m) - and in terms of spectral resolution (hyperspectral imagery). However, the Signal-to-Noise Ratio (SNR) of a Multi-Spectral or Hyper-Spectral Imaging (MSI/HSI) sensor is proportional to the ratio between the sensor area and the number of observed spectral bands. Therefore, to maintain a constant SNR, increasing the number of spectral bands in an hyper-spectral image implies a decrease in spatial resolution. As a consequence, our planet is currently observed by MSI systems having a very good spatial resolution but a low spectral resolution and by HSI systems having a very good spectral resolution but a low spatial resolution.

In remote sensing applied to water color, Sentinel-2 MSI and Sentinel-3 Ocean and Land Color Instrument (OLCI) are extensively used. As shown in Fig. 1-which exhibits the spectral band positions and widths of both instruments - for the considered application, they have 13 - with 10,20 , or $60 \mathrm{~m}$ spatial resolution-and 21

This work is partially funded by CNES within the TOSCA "OSYNICO" project. Experiments presented in this paper were carried out using the CALCULCO computing platform, supported by SCoSI/ULCO.

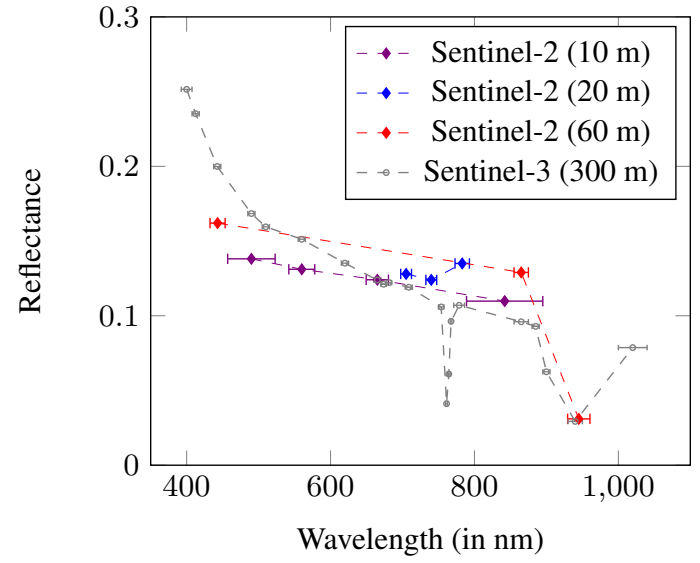

Fig. 1. Examples of Sentinel-2 and Sentinel-3 reflectance spectra.

usable spectral bands-with $300 \mathrm{~m}$ spatial resolution-in the visible and near-infrared range, respectively. Both instruments allow to map several variables describing the biogeochemical dynamics of the marine environment, e.g., Chlorophyll A, Suspended Matter, or Organic Carbon. However, the study of specific environments-such as coastal or estuarine areas-requires observations at a sufficiently fine spatial scale and, at the same time, at a fine spectral resolution, which is not possible using Sentinel-2 or Sentinel-3 alone.

Multi-sharpening [1] or multi-sensor image fusion is the process of combining relevant information from two or more MSI/HSI images into a single image with complementary spatial and spectral resolution characteristics $[1,2]$. To that end, several methods have been proposed in the literature and are based on, e.g., component substitution-using, e.g. Gram-Schmidt [3], the Sylvester equation [4], or Principal Component Analysis [5]—coupled Nonnegative Matrix/Tensor Factorization (NMF/NTF)—e.g., [1, 6, 7]— and more recently on deep learning, e.g., in [8]. However, to the best of the authors knowledge, fusing Sentinel-2 MSI and Sentinel-3 OLCI images was not yet considered in the literature. Indeed, even if in [9], the authors suggested a method for the fusion of data from Sentinel-2 and Sentinel-3, they proposed in practice an approach to combine Sentinel-2 and Moderate Resolution Imaging Spectroradiometer (MODIS) images. The fusion of Sentinel-2 and Sentinel-3 data is let for future work in the conclusion of [9].

Moreover, the authors of [10] considered time series of Sentinel2 and Sentinel-3 images to create daily Sentinel-2 data, i.e., they do not aim to create new multi-spectral images combining the best characteristics of Sentinel-2 and Sentinel-3. On the contrary, they use the same spectral bands in Sentinel-2 and Sentinel-3 in order to generate 
a better spatial resolution of Sentinel-3 images. As a consequence, the objective of these authors slightly differs from ours.

As Sentinel-2 has several bands with coarse to fine spatial resolutions, the super-resolution of Sentinel-2 images has also been considered, e.g., in [11]. The proposed approach is based on a deep convolutional neural network including a residual neural network for the fusion problem modeling. However, taking into account Sentinel-3 data was not considered by the authors of [11].

As a consequence, the goal of this paper is to provide a first comparison of the performance of state-of-the-art multi-sharpening techniques applied to Sentinel-2 and Sentinel-3 data. While an objective comparison of such methods on real data is challengingexcept if we aim to cross-validate their performance, as proposed in, e.g., [12] for HSI unmixing - it remains possible on semi-realistic simulations. We thus aim to propose (i) a first quantitative analysis of the performance of some state-of-the-art techniques on simulated data and (ii) a qualitative analysis of their performance on real data.

The remainder of the paper reads as follows. In Section 2, we briefly introduce the tested methods in this paper. Section 3 (respectively, Section 4) then presents the simulated dataset (respectively, some real dataset) and the reached multi-sharpening performance. Lastly, we conclude and discuss about future work in Section 5.

\section{TESTED MULTI-SHARPENING METHODS}

In this paper, we evaluate the performance of image fusion methods of hyperspectral and multispectral images. To that end, we consider classical and modern multi-sharpening techniques, i.e., (i) based on component substitution-and particularly Gram-Schmidt (GS) [3] and Gram-Schmidt Adaptative (GSA) [13]-(ii) coupled Non-negative Matrix Factorization (NMF) [6], (iii) Fast fusion based on Sylvester equation (FUSE) [4], (iv) Generalized Laplacian pyramid with hypersharpening (GLPHS) [14], (v) Smoothing filter-based intensity modulation with hypersharpening (SFIMHS) [15], (vi) Lanara's work presented in ICCV'15 (ICCV'15) [16], and (vii) Maximum a Posteriori estimation with a Stochastic Mixing Model (MAPSMM) [17]. All these algorithms are provided in the HSMSFusionToolbox ${ }^{1}$ [1]. We decided not to take into account both the "ECCV'14" and "HySure" which were also provided in this toolbox. Indeed, we faced some parameter tuning and execution time issues with the former while the later could not provide any enhancement in our experiments that we do not show because of space constraints. All the tests presented in the remainder of the paper were performed using MATLAB R2019a on Windows 10 x64 machines on a laptop equipped with an Intel i9-9880H CPU @ 2.30GHz with 32.0GB RAM on Windows 10 x64.

The GS transformation is fast and easy to implement and generates fused images with high integration quality color and spatial detail. GS is a commonly used method $[1,3]$ when a low-spatialresolution image is sharpened by adding spatial details obtained by multiplying the difference between a high-spatial-resolution image and a synthetic intensity component by a band-wise modulation coefficient. The improvement lies in computing the synthetic intensity component by performing a linear regression between a highresolution image and lower-resolution bands to mitigate spectral distortion. GSA integrates this technique into the GS algorithm [13]. CNMF [6] unmixes the low-spatial-resolution HSI data and the highspatial-resolution MSI data alternately using the relation between

\footnotetext{
${ }^{1}$ The toolbox may be downloaded at https:// openremotesensing.net/wp-content/uploads/2017/11/ HSMSEusionToolbox.zip.
}

sensor properties for the NMF initializations. CNMF alternately unmixes these two inputs images by NMF to estimate the spectral signatures of endmembers and the high-resolution abundance maps, respectively. CNMF starts by unmixing the low-resolution image using vertex component analysis (VCA) to initialize the endmember signatures. The final high-resolution HSI data is obtained as the product of the spectral signatures and the high-resolution abundance maps. FUSE combines a high-spatial low-spectral resolution image and a low-spatial high-spectral resolution image [4]. FUSE utilizes a Sylvester equation to solve the maximization problem of the likelihoods obtained from the forward observation models. A closed-form solution for the Sylvester equation improved computational performance. In GLPHS fusion, spatial details of each low-resolution band are obtained as the difference between a high-resolution image and its low-pass version multiplied by a global gain factor, which can be computed globally [14]. A Gaussian filter, matching the modulation transfer function (MTF) of a lower-resolution sensor, is used for low pass filtering. SFIMHS [15] is based upon a simplified model for solar radiation and land surface reflection, SFIMHS sharpens the low-resolution image by multiplying an upscaled lower resolution image by a ratio between a higher resolution image and its low-pass filtered image (with a smoothing filter) on a pixel-by-pixel basis [2]. Spatial details can be modulated to a co-registered lower resolution multispectral image without altering its spectral properties and contrast. SFIMHS can be performed on individual HS bands. Similar to CNMF [6], Lanaras's algorithm jointly unmixes the two input images into the spectral signatures of endmembers and (pure reflectance spectra of the observed materials) the associated fractional abundances (mixing coefficients). A projected gradient method was proposed to alternately update the endmember signatures and the high-resolution abundances by solving the two unmixing problems of the input HS-MS images, respectively. Simplex identification via split augmented Lagrangian (SISAL) is used to initialize the endmembers and sparse unmixing by variable splitting and augmented Lagrangian (SUnSAL) is adopted to obtain initial abundances. The formulation leads to a coupled matrix factorization problem, with a number of useful constraints imposed by elementary physical properties of spectral mixing. The MAP-SMM algorithm [17] adopted an SMM to estimate the underlying spectral scene statistics or, more specifically, the conditional mean vector and covariance matrix of the high-resolution HS image with respect to the MS image. The average spectrum, covariance matrix, and abundance map of each endmember are derived from the low-resolution HS image. A MAP objective function is formulated to optimize the high-resolution HS data relative to the input images based on the SMM statistics. The MAP-SMM algorithm is performed in the principal component subspace of the low-resolution HS image.

It is worth mentioning that all the above state-of-the-art methods were proposed for the fusion of two multi- or hyperspectral images with different spatial and spectral resolutions. However, Sentinel-2 MSI data already consist of several spatial resolutions (10,20, and $60 \mathrm{~m}$ ). As a consequence, applying the above multi-sharpening techniques to Sentinel-2 and Sentinel-3 can only be performed for one given Sentinel-2 resolution. As a consequence, we will show below the fusion performance for the three spatial resolutions.

\section{PERFORMANCE OF THE TESTED METHODS ON SYNTHETIC DATA}

Many studies have been published during the last years focusing on single-date, intra-sensor data fusion. As presented in [1,2], the researchers evaluate the performance of fusion algorithms mainly on 
images of the following data sets: AVIRIS Indian Pines, AVIRIS Cuprite, and ROSIS-3 University of Pavia $[1,2]$. In our study, we aim to evaluate the performance of fusion algorithms on Sentinel-2 MSI and Sentinel-3 OLCI remote sensing images. This section thus provides a brief description of the synthetic dataset used in the experiments. To validate the feasibility of Sentinel-3 and Sentinel-2 image fusion, we propose to consider a purely linear model to generate synthetic Sentinel-3 and Sentinel-2 images using AVIRIS ${ }^{2}$ endmembers for Ocean water and Seawater from USGS spectral library ${ }^{3}$ [18]. We then choose 5 endmembers from this library, each of them being related to an ocean or a seawater spectrum. From these endmembers, we extract 21 spectral bands (respectively, 13) which are the closest to Sentinel-3 (respectively, Sentinel-2) ones.

Then, for each endmember, we generate a synthetic abundance map using the Gaussian Fields method [19]. Each of theses maps is of dimension $3000 \times 3000$ and is assumed to have a $10 \mathrm{~m} \mathrm{spa}$ tial resolution. Combining them with the above endmembers allows generate three high-resolution Sentinel-3-like images, i.e., a $3000 \times 3000 \times 21$ datacube. We then apply a Gaussian filter to downsample the spatial content and we derive both a $1500 \times 1500 \times 21$ and a $500 \times 500 \times 21$ datacubes that we aim to estimate from their downsampled versions. We then generate the observed data by (i) downsampling — by a factor 30 - the $3000 \times 3000$ maps by using a Gaussian filter, in order to derive the observed $100 \times 100 \times 21$ synthetic Sentinel-3, with $300 \mathrm{~m}$ spatial resolution. We then derive the observed synthetic Sentinel-2 datacube by applying to the $3000 \times 3000$ abundance maps a downscale factor of two (with $20 \mathrm{~m}$ spatial resolution) and six (with $60 \mathrm{~m}$ spatial resolution) and by considering the corresponding spectral bands used in the real Sentinel-2 imagers, i.e., four bands with $10 \mathrm{~m}$, six bands with $20 \mathrm{~m}$, and three bands with $60 \mathrm{~m}$ spatial resolution of Sentinel-2.

In order to assess the performance of the tested methods, we use some classical quantitative performance measures $[1,20]$, i.e., (i) the Peak Signal-to-Noise Ratio (PSNR) - which is the ratio between the highest possible signal energy and the noise energy-(ii) the Spectral Angle Mapper (SAM) - which is a pixelwise measure of the angle between the reference spectrum and the fused one. SAM values near zero indicate local high spectral quality and we use the average SAM value with respect to pixels for the quality index of the entire data set-(iii) the ERGAS measure-i.e., a normalized average error of each band of processed image - and (iv) Q2n which is a generalization of the universal image quality index (UIQI) and an extension of the Q4 index to HS images based on hypercomplex numbers [1].

As already explained, the tested state-of-the-art methods are designed to fuse two datacubes with two distinct resolutions. However, Sentinel-2 already has 3 different spatial resolution at different wavelengths. One must thus choose a target spatial resolution among the three available, i.e., 10,20 , or $60 \mathrm{~m}$. Table 1 shows the obtained results for each of these resolutions. They show that FUSE is almost always outperforming the other techniques. Then-except for $60 \mathrm{~m}$ resolution where GLPHS outperforms FUSE-GLPHS, MAPSMM, and SFIMHS provide some performance slightly lower than FUSE. Lastly, the other tested methods provide some performance significantly lower than the former. Let us emphasize again that these results were obtained with simulations in which the purely linear mixing model is assumed. However, such an assumption might not be satisfied in practice.

\footnotetext{
${ }^{2}$ See https://aviris.jpl.nasa.gov/.

${ }^{3}$ This library is accessible at https://crustal.usgs.gov/ speclab/AV14.php.
}

\begin{tabular}{|c||c|c|c|c|}
\hline Method & PSNR & SAM & ERGAS & Q2n \\
\hline \hline \multicolumn{5}{|c|}{ Perf. obtained with 60 m spatial resolution } \\
\hline \hline GSA & 11.3 & 11.8811 & 8.969 & $\mathbf{1}$ \\
\hline FUSE & 23.5 & 0.26654 & 1.6697 & $\mathbf{1}$ \\
\hline CNMF & 15.3 & 0.49605 & 4.3611 & $\mathbf{1}$ \\
\hline GLPHS & $\mathbf{2 3 . 7}$ & $\mathbf{0 . 2 6 2 0 8}$ & $\mathbf{1 . 6 3 2 9}$ & $\mathbf{1}$ \\
\hline SFIMHS & 22.8 & 0.28683 & 1.8134 & $\mathbf{1}$ \\
\hline ICCV15 & 18.8 & 0.39035 & 2.8472 & $\mathbf{1}$ \\
\hline MAP-SMM & 23.2 & 0.28777 & 1.7412 & $\mathbf{1}$ \\
\hline \hline \multicolumn{5}{|c|}{ Perf. obtained with 20 m spatial resolution } \\
\hline \hline \multicolumn{1}{|c||}{ GSA } & 9.8 & 9.4837 & 2.9385 & $\mathbf{1}$ \\
\hline FUSE & $\mathbf{2 1 . 6}$ & $\mathbf{0 . 3 4 3 5 9}$ & $\mathbf{0 . 7 0 2 2 4}$ & $\mathbf{1}$ \\
\hline CNMF & 8.4 & 0.47334 & 3.4464 & 0.67949 \\
\hline GLPHS & 21.4 & 0.36691 & 0.71696 & $\mathbf{1}$ \\
\hline SFIMHS & 21.1 & 0.35972 & 0.74190 & $\mathbf{1}$ \\
\hline ICCV15 & 18.2 & 0.45740 & 1.0388 & $\mathbf{1}$ \\
\hline MAP-SMM & 21.0 & 0.36554 & 0.75128 & $\mathbf{1}$ \\
\hline \hline \multicolumn{6}{|c||}{ Perf. obtained with $10 \mathrm{~m}$ spatial resolution } \\
\hline \hline GSA & 17.4 & 0.8112 & 0.56697 & 0.99774 \\
\hline FUSE & $\mathbf{1 9 . 9}$ & $\mathbf{0 . 4 3 2 1}$ & $\mathbf{0 . 4 2 8 6 9}$ & $\mathbf{1}$ \\
\hline CNMF & 15.2 & 0.60021 & 0.74173 & $\mathbf{1}$ \\
\hline GLPHS & 19.3 & 0.48046 & 0.46072 & $\mathbf{1}$ \\
\hline SFIMHS & 19.6 & 0.44427 & 0.44506 & $\mathbf{1}$ \\
\hline ICCV15 & 17.3 & 0.55533 & 0.57638 & $\mathbf{1}$ \\
\hline MAP-SMM & 19.2 & 0.43245 & 0.44757 & $\mathbf{1}$ \\
\hline
\end{tabular}

Table 1. Performance of the fusion methods on simulations.

\section{PERFORMANCE OF THE TESTED METHODS ON REAL DATA}

We now investigate the performance reached by the tested methods on real Sentinel-2 and Sentinel-3 datasets ${ }^{4}$. The selected study area is located in the islands of The Bahamas in the Atlantic Ocean. The Tongue of the Ocean is a deep-water basin in the Bahamas that is surrounded to the East, West, and South by a carbonate bank known as the Great Bahama Bank. The deep blue water of the Tongue is a stark contrast to the shallow turquoise waters of the surrounding Bank. Generally, waters that are optically shallow (e.g., Grand Bahama Bank) appear blue-green due to high bottom reflectance contributions while optically deep waters appear dark blue. The centre of the Bahamas image is located at the following coordinates: Lat: 26 35'58.50”'N, and Lon: $77^{\circ} 28^{\prime} 29.93$ ”'W (DMS), Projection UTM, Zone $18 \mathrm{~N}$, and World Geodetic System 1984.

To assess the performance of the tested methods, as we do not have any ground truth information, we propose to compare their multi-sharpening outputs with those obtained using the ENVI implementation of GS [3]. To do so, we use the same performance criteria as in the previous section. However, these results must be carefully interpreted. Indeed, a good performance index-i.e., a high PSNR, a low SAM, a low ERGAS or a high Q2n-only indicates that the fusion result obtained with a tested method is close to the one reached by GS. On the contrary, the fact that one performance index is "poor" only states that the reached performance is different from GS. At this stage, we are not able to decide whether the performance is "better" or "worse" than with GS. Table 2 summarizes the obtained results. One may see that, for each given spatial resolu-

\footnotetext{
${ }^{4}$ They are publicly available at https://scihub.copernicus. eu/dhus/\#/home.
} 


\begin{tabular}{|c|c|c|c|c|}
\hline Method & PSNR & SAM & ERGAS & Q2n \\
\hline \multicolumn{5}{|c|}{$\begin{array}{l}\text { Perf. obtained with } 60 \mathrm{~m} \text { spatial resolution } \\
\end{array}$} \\
\hline GSA & 26.7 & 0.2518 & 20.9157 & 0.88583 \\
\hline FUSE & 25.8 & 0.2154 & 19.8935 & 0.81807 \\
\hline CNMF & 27.5 & 0.2046 & 16.7684 & 0.91749 \\
\hline GLPHS & 25.6 & 0.2166 & 21.3205 & 0.93843 \\
\hline SFIMHS & 25.6 & 0.2197 & 21.7276 & 0.87596 \\
\hline ICCV15 & 28.5 & 0.1887 & 14.054 & 0.95898 \\
\hline MAP-SMM & 25.6 & 0.2196 & 21.48 & 0.91038 \\
\hline \multicolumn{5}{|c|}{ Perf. obtained with $20 \mathrm{~m}$ spatial resolution } \\
\hline GSA & 23.9 & 0.2427 & 5.2917 & 0.86474 \\
\hline FUSE & 18.8 & 0.2500 & 5.6916 & 0.80446 \\
\hline CNMF & 21.7 & 0.2570 & 6.9271 & 0.75592 \\
\hline GLPHS & 23.2 & 0.2406 & 5.8331 & 0.90253 \\
\hline SFIMHS & 23.0 & 0.2420 & 5.9424 & 0.86716 \\
\hline ICCV15 & 23.3 & 0.2278 & 5.6937 & 0.93979 \\
\hline MAP-SMM & 23.1 & 0.2465 & 5.8853 & 0.85204 \\
\hline \multicolumn{5}{|c|}{ Perf. obtained with $10 \mathrm{~m}$ spatial resolution } \\
\hline 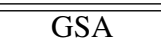 & 24.3 & $\overline{0.2550}$ & 2.6741 & "0.89361 \\
\hline FUSE & 21.4 & 0.2596 & 2.7337 & 0.86143 \\
\hline CNMF & 23.9 & 0.2492 & 2.7735 & 0.92034 \\
\hline GLPHS & 23.3 & 0.2495 & 2.9307 & 0.86243 \\
\hline SFIMHS & 23.2 & 0.2521 & 2.9953 & 0.86188 \\
\hline ICCV15 & 23.9 & 0.2289 & 2.6807 & 0.92013 \\
\hline MAP-SMM & 14.7 & 0.2008 & 1.3722 & 0.99306 \\
\hline
\end{tabular}

Table 2. Reached performance wrt GS output on real data.

tion, all the tested methods (except MAP-SMM for $10 \mathrm{~m}$ of spatial resolution) provide a quite similar performance with respect to these indices. We thus can state that they globally provide a quite similar performance. However, when we visually look at the fused images, one might see some specific behaviors, as we now discuss.

Due to space constraints, we only show the performance reached for $60 \mathrm{~m}$ of spatial resolution. As the tested methods generate some datacubes which are not easy to draw in a compact way, we propose the following procedure. For each pixel, we average the amplitudes along the spectrum axis. We then derive an image that we draw according to the same scale. All these images are shown in Fig. 2. In particular, Fig. 2(a) represents the original $1830 \times 1830 \times 3$ Sentinel2 image that we aim to fuse with the $366 \times 366 \times 16$ Sentinel- 3 one plot in Fig. 2(b). Figures 2(c) to 2(i) show the obtained fused images with the different tested methods. One may notice that most of them provide some artefacts. In particular, FUSE seems to provide the worst visual performance. Indeed, one notice some visible artefacts providing a kind of texture. Moreover, it also provides some negative amplitudes which have no physical meaning. Then, please notice that the Sentinel-3 image seem to provide more spatial information that the Sentinel-2 one. This is probably due to the fact that, even if both images were taken the same day, they were not taken at the same time and that the considered Sentinel-2 data only contain 3 spectral samples. However, CNMF and GSA seem to provide a higher visual quality than the other methods. Both methods can also partially remove clouds from Sentinel-3 images. Then, both SFIMHS and MAP-SMM provide some similar images, with a visually good spatial information. Lastly, ICCV'15 seems to stick to Sentinel-2 spatial information. However, this is not the case when the spatial resolution is $10 \mathrm{~m}$-not shown for space considerationwhere it provides the best visual information. Unfortunately, in the absence of ground truth, it is hard to provide more information about these results, which is a perspective discussed in the next section.

\section{CONCLUSION AND PERSPECTIVES}

In this paper, we presented a first comparative study of Sentinel-2 and Sentinel-3 image fusion methods. As the ratio between high and low spatial resolutions increases, and for higher spatial resolution $(10 \mathrm{~m})$, the fusion performance decreases. According to the considered experiments, CNMF and ICCV' 15 seem to be the more robust tested methods. In future work, we aim to propose a new method able to take into account all the bands of Sentinel-2 in order to provide a new multi-spectral image with 10 -m spatial resolution and Sentinel-3 spectral bands. Moreover, we aim to compare the above methods and our future work with in situ measurements. To that end, atmospheric correction [21] will be applied to Sentinel-2 and 3 data.

\section{REFERENCES}

[1] N. Yokoya, C. Grohnfeldt, and J. Chanussot, "Hyperspectral and multispectral data fusion: A comparative review of the recent literature," IEEE Geosci. Remote Sens. Mag., vol. 5, no. 2, pp. 29-56, 2017.

[2] L. Loncan, L. B. De Almeida, J. M. Bioucas-Dias, X. Briottet, J. Chanussot, N. Dobigeon, S. Fabre, W. Liao, G. A. Licciardi, M. Simoes, et al., "Hyperspectral pansharpening: A review," IEEE Geosci. Remote Sens. Mag., vol. 3, no. 3, pp. 27-46, 2015.

[3] C. A. Laben and B. V. Brower, "Process for enhancing the spatial resolution of multispectral imagery using pan-sharpening," 2000, US Patent 6,011,875.

[4] Q. Wei, N. Dobigeon, and J.-Y. Tourneret, "Fast fusion of multi-band images based on solving a sylvester equation," IEEE Trans. Image Process., vol. 24, no. 11, pp. 4109-4121, 2015.

[5] P. Kwarteng and A. Chavez, "Extracting spectral contrast in landsat thematic mapper image data using selective principal component analysis," Photogramm. Eng. Remote Sens, vol. 55, no. 1, pp. 339-348, 1989.

[6] N. Yokoya, T. Yairi, and A. Iwasaki, "Coupled nonnegative matrix factorization unmixing for hyperspectral and multispectral data fusion," IEEE Trans. Geosci. Remote Sens., vol. 50, no. 2, pp. 528-537, 2011.

[7] S. Li, R. Dian, L. Fang, and J. M. Bioucas-Dias, "Fusing hyperspectral and multispectral images via coupled sparse tensor factorization," IEEE Trans. Image Process., vol. 27, no. 8, pp. 4118-4130, 2018.

[8] C. Dong, C. C. Loy, K. He, and X. Tang, "Image superresolution using deep convolutional networks," IEEE Trans. Pattern Anal. Mach. Intell., vol. 38, no. 2, pp. 295-307, 2015.

[9] A. Korosov and D. Pozdnyakov, "Fusion of data from Sentinel2/MSI and Sentinel-3/OLCI," in Living Planet Symposium, 2016, vol. 740, p. 405.

[10] Q. Wang and P. M. Atkinson, "Spatio-temporal fusion for daily sentinel-2 images," Remote Sensing of Environment, vol. 204, pp. 31-42, 2018.

[11] F. Palsson, J. R. Sveinsson, and M. O. Ulfarsson, "Sentinel-2 image fusion using a deep residual network," Remote Sensing, vol. 10, no. 8, pp. 1290, 2018. 


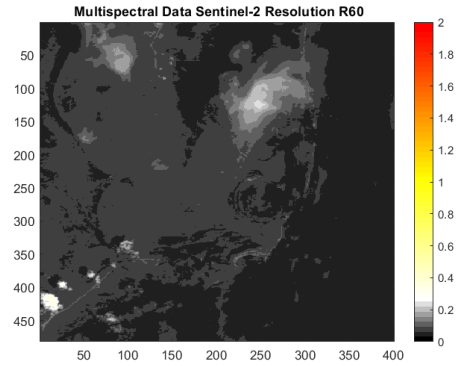

(a)

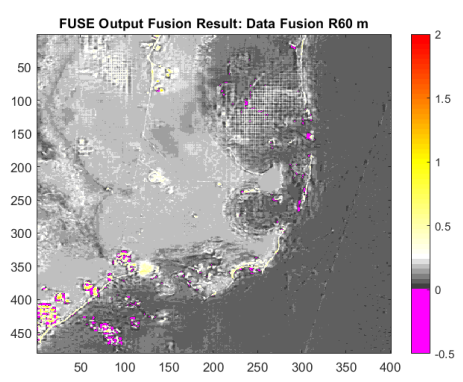

(d)

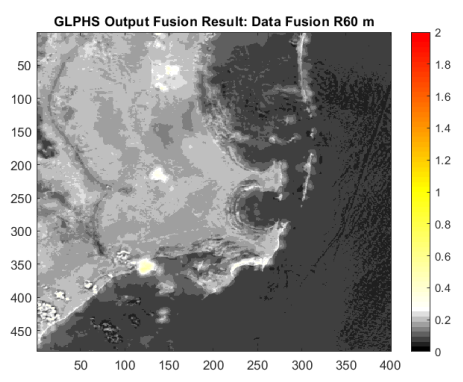

(g)

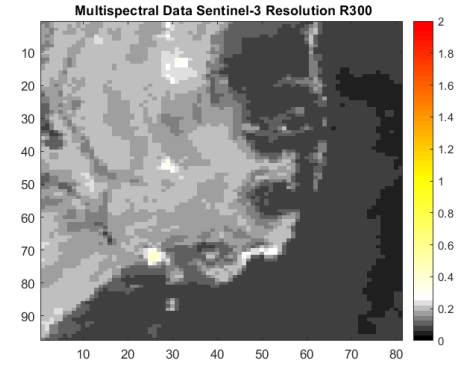

(b)

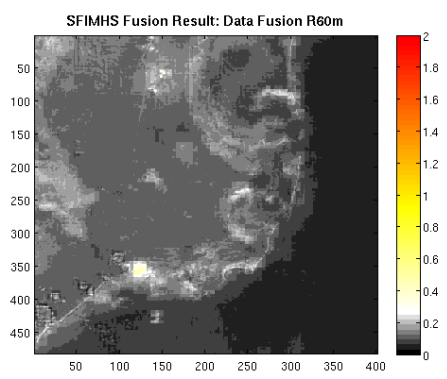

(e)

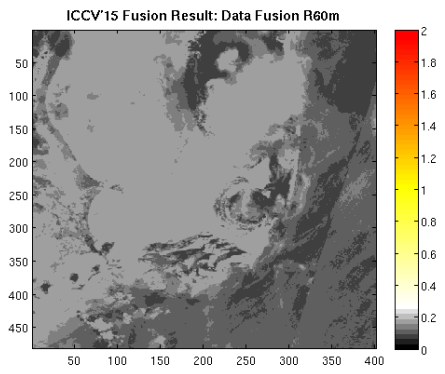

(h)

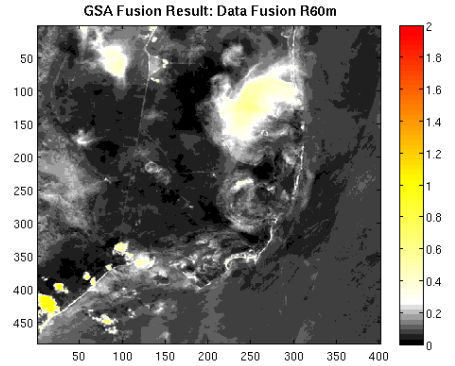

(c)

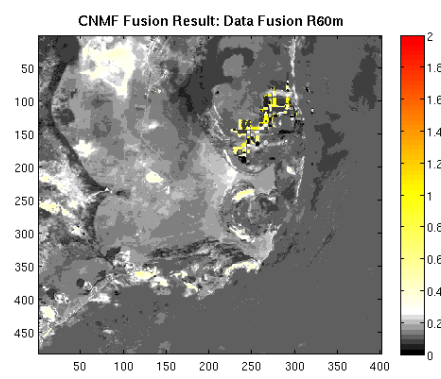

(f)

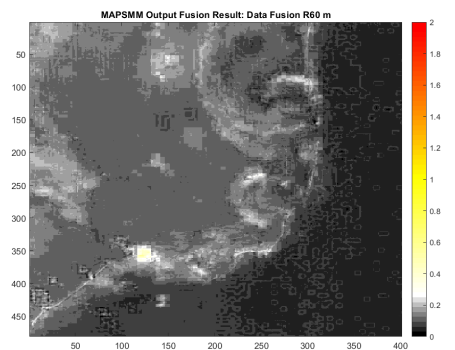

(i)

Fig. 2. Fused Sentinel-2 (with $60 \mathrm{~m}$ spatial resolution) and Sentinel-3 images, with several methods: (a) original Sentinel-2 image, (b) original Sentinel-3 image, (c) GSA, (d) FUSE, (e) SFIMHS, (f) CNMF, (g) GLPHS, (h) ICCV’15, (i) MAP-SMM.

[12] M. Puigt, O. Berné, R. Guidara, Y. Deville, S. Hosseini, and C. Joblin, "Cross-validation of blindly separated interstellar dust spectra," in Proc. ECMS'09, 2009, pp. 41-48.

[13] B. Aiazzi, S. Baronti, and M. Selva, "Improving component substitution pansharpening through multivariate regression of ms + pan data," IEEE Trans. Geosci. Remote Sens., vol. 45, no. 10, pp. 3230-3239, 2007.

[14] B. Aiazzi, L. Alparone, S. Baronti, A. Garzelli, and M. Selva, "MTF-tailored multiscale fusion of high-resolution MS and pan imagery," Photogrammetric Engineering \& Remote Sensing, vol. 72, no. 5, pp. 591-596, 2006.

[15] J. Liu, "Smoothing filter-based intensity modulation: A spectral preserve image fusion technique for improving spatial details," International Journal of Remote Sensing, vol. 21, no. 18, pp. 3461-3472, 2000.

[16] C. Lanaras, E. Baltsavias, and K. Schindler, "Hyperspectral super-resolution by coupled spectral unmixing," in Proc. IEEE ICCV'15, 2015, pp. 3586-3594.
[17] M. T. Eismann, Resolution enhancement of hyperspectral imagery using maximum a posteriori estimation with a stochastic mixing model, Ph.D. thesis, University of Dayton, 2004.

[18] R. F. Kokaly, R. N. Clark, G. A. Swayze, K. E. Livo, T. M. Hoefen, N. C. Pearson, R. A. Wise, W. M. Benzel, H. A. Lowers, R. L. Driscoll, et al., "USGS spectral library version 7," Tech. Rep., US Geological Survey, 2017.

[19] Grupo de Inteligencia Computacional, UPV/EHU, "Hyperspectral imagery synthesis (EIAs) toolbox," http: / / www . ehu.es/ccwintco/index.php/Hyperspectral_ Imagery_Synthesis_tools_for_MATLAB.

[20] P. Jagalingam and A. V. Hegde, "A review of quality metrics for fused image," Aquatic Procedia, vol. 4, pp. 133-142, 2015.

[21] F. Steinmetz and D. Ramon, "Sentinel-2 MSI and sentinel-3 OLCI consistent ocean colour products using POLYMER," in Proc. SPIE "Remote Sensing of the Open and Coastal Ocean and Inland Waters", 2018, vol. 10778. 